\title{
$\begin{array}{ll}\text { Research Square } & \text { Preprints are preliminary reports that have not undergone peer review. } \\ \text { They should not be considered conclusive, used to inform clinical practice, } & \text { or referenced by the media as validated information. }\end{array}$
}

\section{Ascorbic Acid as an Adjunctive Therapy in Critically III Patients with COVID-19: A Multicenter Propensity Score Matched Study}

Khalid Al Sulaiman ( $\nabla$ alsulaimankh@hotmail.com )

King Abdulaziz Medical City

Ohoud Al Juhani

King Abdulaziz University

Khalid Bin Salah

King Abdulaziz Medical City

Hisham A. Badreldin

King Abdulaziz Medical City

Abdullah Al Harthi

King Abdulaziz Medical City

Mohammed Alenazi

King Saud bin Abdulaziz University for Health Sciences

Aisha Alharbi

King Abdulaziz University Hospital

Rahmah Algarni

King Abdulaziz University Hospital

Shmeylan Al Harbi

King Abdulaziz Medical City

Abdullah Alhammad

King Saud University

Ramesh Vishwakarma

King Abdullah International Medical Research Center

Sarah Aldekhyl

King Abdulaziz Medical City

\section{Research Article}

Keywords: COVID-19, SARS-CoV-2, Ascorbic Acid, Vitamin C, Vitamins, Critically ill, Intensive Care Units (ICUs), 30-days ICU mortality

Posted Date: April 2nd, 2021 
DOl: https://doi.org/10.21203/rs.3.rs-354711/v1

License: (c) (1) This work is licensed under a Creative Commons Attribution 4.0 International License. Read Full License 


\section{Abstract}

\section{Background:}

Ascorbic acid represents an appealing option for clinicians to utilize in the context of a global health pandemic of COVID-19 patients due to its proposed clinical efficacy, relative safety, and low cost.

\section{Objectives:}

The aim of this study was to evaluate the efficacy and safety of using ascorbic acid in supplemental dose as adjunctive therapy in critically ill patients with COVID-19.

\section{Methods:}

This was a multi-center, non-interventional, retrospective cohort study. All critically ill adult patients admitted to ICU with a confirmed COVID-19 between March 1st to December 31 $1^{\text {st }}, 2020$ were included in the final analysis. The study was conducted at two large governmental tertiary hospitals in Saudi Arabia. The purpose was to investigate the association between clinical outcomes with ascorbic acid use as an adjunctive therapy in COVID-19 after propensity score matching using baseline severity scores, systemic use of corticosteroids and study centers.

\section{Results:}

A 739 patients were included in this study; 296 patients were included after propensity score matching. There was no association between the administration of ascorbic acid and in-hospital mortality nor 30day ICU mortality (OR $(95 \% \mathrm{Cl}): 0.77(0.476,1.234)$, p-value $=0.2738$ and OR $(95 \% \mathrm{Cl}): 0.73(0.438,1.204)$, $p$-value $=0.215$ respectively). Using ascorbic acid was associated with lower incidence of thrombosis compared with the non-ascorbic acid group (6.1\% vs. $13 \%$ respectively); OR $(95 \% \mathrm{Cl}): 0.42(0.184,0.937)$, p-value $=0.0342$ ).

\section{Conclusion:}

Supplemental dose of ascorbic acid as an adjunctive therapy in COVID19 critically ill patients was not associated with mortality benefits; but associated with lower incidence of thrombosis. Further studies are required to confirm these findings.

\section{Introduction}

Coronavirus Disease 2019 (COVID-19) and its related Severe Acute Respiratory Syndrome Coronavirus-2 (SARS-CoV-2) represents one of the most recent serious healthcare challenges known to the mankind. To date, most of the available investigated treatments are supportive measures with few proposed preventive measures. ${ }^{1}$ There are several agents proposed to have a role in treatment and prevention of COVID-19. Due to its known antioxidant effects and role in enhancing immune function, ascorbic acid 
(Vitamin C) was assumed to have beneficial effect in COVID-19. This is mainly via supporting lymphocyte activity, stimulating interferon-a production, reducing inflammation, and improving endothelial function; thus, it might exert an antiviral effect ${ }^{2-4}$

Ascorbic acid is a water-soluble vitamin that is believed to have clinical benefits for patients with severe illnesses. Due to its antioxidant properties, it has been evaluated in severe oxidative stress status such as serious infection, sepsis and ARDS. COVID-19 infections can lead to serious oxidative stress leading to a state where patients might require more ascorbic acid. Several reports addressed ascorbic acid's potential effect in ameliorating the inflammation and vascular injury in critically ill patients. ${ }^{5,6}$ In light of its proposed efficacy, relative safety, and low cost, ascorbic acid represents an appealing agent for researchers and clinicians to utilize in the context of a global health pandemic.

There are several studies with mixed results regarding the clinical use of ascorbic acid in non-COVID-19 critically ill patients. A pilot study compared intravenous (IV) ascorbic acid in two different regimens with placebo arm in 24 critically ill patients with sepsis. This study showed that patients who received IV ascorbic acid had lower sequential organ failure assessment (SOFA) scores and lower levels of proinflammatory markers compared to the placebo group. ${ }^{7}$ Another randomized controlled study conducted in 167 critically ill patients with sepsis induced ARDS found no difference in SOFA scores and levels of inflammatory markers between the groups. However, the 28-day mortality was lower in the treatment group. ${ }^{8}$

Ascorbic acid and thamine levels are low in sepsis. Thiamine modifies the metabolism of ascorbic acid leading the decrease in oxalate production, thereby decreasing the likelihood of oxalate nephropathy. Hydrocortisone is well studied in sepsis and may work synergistically with ascorbic acid ${ }^{9,24,32}$. Therefore, ascorbic acid has also been evaluted in some studies as part of a combination regimen with thiamine and hydrocortisone ${ }^{9,24}$. There were two small retrospective studies that found some improvement in the clinical outcomes (i.e., reduced mortality, reduced risk of progression to organ failure, and improved radiographic findings) in patients with sepsis or severe pneumonia who received ascorbic acid as part of combination regimen with thiamine, and hydrocortisone ${ }^{9,10}$. One meta analysis evaluted ascorbic acid use in intensive care unit (ICU) patients without COVID-19 found that high-dose intravenous ascorbic acid infusions (i.e., $200 \mathrm{mg} / \mathrm{kg} /$ day) shortened the ICU length of stay by $7.8 \% .{ }^{11} \mathrm{~A}$ similar experiment was conducted in critically ill patients with severe influenza in which ascorbic acid was shown to reduce oxidative stress related lung injury. ${ }^{12,13} \mathrm{~A}$ recent report that invistigated the use of high-dose intravenous ascorbic acid in treatment of 50 moderate to severe COVID-19 patients, showed an improvement in the oxygenation index. ${ }^{28}$

Overall, there is limited data to support the use of ascorbic acid in critically ill patients with COVID-19. In this context, this study aims to investigate the safety and efficacy of ascorbic acid use as adjunctive therapy in COVID-19 critically ill patients. 


\section{Methods}

\section{Study design:}

This was a multi-center, observational study with retrospective analysis of prospectively collected data in consecutive critically ill patients who admitted to ICUs with a confirmed diagnosis of COVID-19 in Saudi Arabia. The diagnosis of COVID-19 was confirmed by Reverse Transcriptase - Polymerase Chain Reaction (RT-PCR) on nasopharyngeal and/or throat swabs. All the patients who met our inclusion criteria during the study period $(01 / 03 / 2020-31 / 12 / 2020)$ were included. Included patients were divided into 2 groups based on ascorbic acid use as adjunctive therapy during ICU stay. All patients were followed until they were discharged from the hospital or died during in-hospital stay whichever occurred first. The study was approved by the King Abdullah International Medical Research Center (KAIMRC)/Institutional Review Board (IRB), Riyadh, Saudi Arabia (Study Number: RC20/589/R). KAIMRC-IRB committee waived the informed consent due to its retrospective nature.

Propensity score matching Procedure (Proc PS match) (SAS, Cary, NC) was used to match patients who received ascorbic acid to patients who did not. A greedy nearest neighbor matching method was used in which one non ascorbic acid (control) is matched with each patient in the ascorbic acid (active) group which eventually produced the smallest within-pair difference among all available pairs with treated patients. Patients were matched only if the difference in the logits of the propensity scores for pairs of patients from the two groups was less than or equal to 0.5 times the pooled estimate of the standard deviation.

\section{Eligibility criteria:}

Adults' patients ( $\geq 18$ years old) were enrolled in the study if they were admitted to the ICU with confirmed diagnosis of COVID-19 using the PCR test. Patients were excluded if the ICU length of stay (LOS) was less than 24 hours or more than 60 days, and/or labeled as "Do-Not-Resuscitate" status within 24 hours of ICU admission.

\section{Setting:}

This study was conducted in two tertiary governmental hospitals; King Abdulaziz Medical City, Riyadh and King Abdulaziz University Hospital, Jeddah. The primary site for this multicenter study was King Abdulaziz Medical City (Riyadh).

\section{Data collection:}

The following information were collected: demographic data (See additional file-1), comorbidities, vital signs and laboratory tests, severity baseline scores (i.e. Acute Physiology And Chronic Health Evaluation II (APACHE II), Sequential Organ Failure Assessment (SOFA) and Nutrition Risk in Critically ill (NUTRIC)), Glasgow Coma Score (GCS), acute kidney injury (AKI), fluid balance, needs for mechanical ventilation (MV) and MV settings within 24 hours of ICU admission. Additionally, renal profile, liver function tests 
(LFTs), coagulation profile (i.e., INR, aPTT, fibrinogen), and inflammatory markers (CRP, procalcitonin) within 24 hours of ICU admission were collected. Lastly corticosteroids and tocilizumab use were recorded for the eligible patients and followed due to their potential benifts.

\section{Endpoints:}

The primary endpoint was estimating the in-hospital mortality in critically ill patients with COVID-19 who received supplemental dose of ascorbic acid as adjunctive therapy versus those who didn't receive ascorbic acid. The secondary endpoints were the following, 30-days ICU mortality, ICU LOS, hospital LOS, MV duration. We also reported the following complications during ICU stay: AKI, liver injury, respiratory failure, thrombosis and infraction.

\section{Definition (s)}

- Acute kidney injury (AKI was defined using Acute Kidney Injury Network (AKIN) definition ${ }^{29}$, which is a sudden decrease of renal function within 48 hours, defined by an increase in absolute $\mathrm{SCr}$ of at least $26.5 \mu \mathrm{mol} / \mathrm{L}(0.3 \mathrm{mg} / \mathrm{dL})$ or by a percentage increase in $\mathrm{SCr} \geq 50 \%(1.5 \times$ baseline value), or by a decrease in the urine output (UOP) (documented oliguria $<0.5 \mathrm{~mL} / \mathrm{kg} / \mathrm{h}$ for more than 6 hours).

- Liver injury was defined as alanine aminotransferase (ALT) exceeding 3 times the upper limit of normal or double in patients with elevated baseline ALT during hospital stay.

- Respiratory failure was defined as either hypoxemic respiratory failure $\left(\mathrm{PaO}_{2}<60 \mathrm{~mm} \mathrm{Hg}\right.$ with a normal or low arterial carbon dioxide tension $\left(\mathrm{PaCO}_{2}\right)$ or hypercapnic respiratory failure $\left(\mathrm{PaCO}_{2}>50\right.$ $\mathrm{mm} \mathrm{Hg}$ ) that requires invasive mechanical ventilation.

- Thrombosis/infraction was defined using the International Classification of Diseases, Tenth Revision, Clinical Modification (ICD10-CM) code (i.e. myocardial infarction (MI), ischemic stroke, pulmonary embolism, deep vein thrombosis) during ICU stay ${ }^{30}$.

\section{Statistical analysis:}

Categorical variables were reported using numbers and percentages. Continuous variables were reported using mean with standard deviation (SD), or median with interquartile range (IQR) when appropriate. We compared categorical variables using the Chi square or Fisher exact test. Continuous variables were compared using numerical the student-t test (for the normally distributed variables) or other quantitative variables with the Mann-Whitney $U$ test (for the non-normally distributed variables). The normality assumptions were assessed for all numerical variables using statistical test (i.e., Shapiro-Wilk test) and also using graphical representation (i.e., histograms and Q-Q plots). A Kaplan-Meier plot was used to describe survival rates and compared using log rank test. Baseline characteristics, baseline severity and endpoints variables were compared between the two treatment groups. Multivariate logistic regression and generalized linear regression were used to find out the relationship between ascorbic acid use and 
different outcomes considered in this study. We considered a P value of $<0.05$ statistically significant and used SAS version 9.4 for all statistical analyses.

We assessed model fit using the Hosmer-Lemeshow goodness-of-fit test. The odds ratios (OR) and estimates with the $95 \%$ confidence intervals $(\mathrm{Cl})$ were reported for the associations. No imputation was made for missing data as the cohort of patients in our study was not derived from random selection.

\section{Results}

A total of 739 patients met the inclusion criteria. Of those included, 158 (21.3\%) patients received ascorbic acid whereas $581(78.7 \%)$ patients did not. A total of 296 patients were included after propensity score matching using baseline severity scores (i.e., APACHE II, SOFA score, NUTRIC scores), systemic use of corticosteroids and study centers ${ }^{31}$.

We observed that all included patients in the ascorbic acid group received a fixed dose of $1000 \mathrm{mg}$ of ascorbic acid enterally once daily with a median duration of administration of 11 days (IQR 7-18). Ascorbic acid was initiated within 24 hours of ICU admission in (61.9\%) of the patients.

\section{Demographic and Clinical Characteristics}

The majority of the included patients in both arms were male (72\%) with a mean age of 60.65 (SD +14.81) (Additional file 1). The predominant underlying comorbidities were diabetes mellitus (59\%) followed by hypertension (56\%) and dyslipidemia (29\%). Most of the comorbidities were similar between the two groups (Additional file 2).

Patients who didn't receive ascorbic acid as adjunctive therapy had higher baseline severity scores (i.e., APACHE II, SOFA, and NUTRIC scores), AKI, requiring MV within 24 hours of ICU admission, and higher baseline laboratory tests (Additional file 1). Conversely, patients who received ascorbic acid as adjunctive therapy had significantly higher systemic corticosteroid use during ICU, estimated glomerular filtration rate (eGFR), and pH. Following the propensity score matching, most of these baseline and demographic characteristics were shown to be similar between the two groups. (Additional file 1).

\section{Endpoints}

During the hospital stay, the analysis for all eligible patients who received ascorbic acid showed significantly lower in-hospital mortality rate in comparison to the non-ascorbic acid group (33.6\% vs. $49.3 \%$ respectively, $P=0.0006$ ). However, after propensity matching, the difference between the two groups became statistically insignificant (32.4\% in the ascorbic acid groups vs. $41.6 \%$ in the non-ascorbic acid group, $\mathrm{P}=0.11$ ). (Table 1 ). As shown in the Kaplan-Meier curve, the overall survival probability during hospital stay was statistically significant before propensity score matching among patients who used ascorbic acid; however, after propensity score matching there was no significant difference. (Figure 1a, 1b). 
Among those who survived during ICU stay, we observed that critically ill patients who received a supplemental dose of ascorbic acid as adjunctive therapy had a longer ICU LOS, and hospital LOS with a beta coefficient $(95 \% \mathrm{Cl}): 0.47(0.26,0.68)$, p-value $<0.0001$, and beta coefficient $(95 \% \mathrm{Cl}): 0.50(0.29,0.71)$, p-value $<0.0001$ respectively (Table 1 ).

\section{ICU Complications during ICU stay}

We observed that the ascorbic acid group was shown to have statistically insignificant higher rate of AKI, liver Injury and respiratory failure requiring MV compared with the non-ascorbic acid group. (Table 2).

On the other hand, we observed that the ascorbic acid group had statistically significant lower rate of thrombosis/infraction compared with the non-ascorbic acid group ( $6.1 \%$ vs $13 \%$ respectively); (OR (95\% Cl): 0.42 (0.184, 0.937), p-value=0.03) (Table 2).

\section{Discussion}

In this retrospective cohort study of critically ill patients with COVID-19, patients who received enteral ascorbic acid in a dose of $1000 \mathrm{mg}$ daily (supplemental dose) have similar mortality rate with patients who did not receive it.

We observed that both the in-hospital and 30-days ICU mortality were similar in both groups after matching patients based on the severity of illness (i.e., APACHE II, SOFA score, NUTRIC scores), study center, and steroid use. More patients in the ascorbic acid group received a systemic steroid in our study than in the non-ascorbic acid group 92.9 vs. 86.5 (P-value 0.0312 ), respectively. This could justify ascorbic acid's survival benefit before controlling for the effect of steroid use in the overall cohort. However, the ascorbic acid group showed no mortality benefits after controlling for steroid use's potential impact.

In critically ill patients, the ascorbic acid deficiency is commonly observed despite receiving proper ascorbic acid intake ${ }^{(16)}$. Furthermore, in critically ill patients, the ascorbic acid deficiency is associated with multi-organ failure and increased mortality $(17,18)$. A bioinformatic study highlighted the potential role of ascorbic acid in sepsis. By suppressing inflammatory response and oxidative stress, which are vital pathophysiological mechanisms of sepsis, ascorbic acid may have a beneficial effect against sepsis ${ }^{(19)}$. Moreover, patients with severe COVID-19 are known to have higher inflammatory markers and cytokine storm ${ }^{31}$.

Large randomized controlled studies using ascorbic acid for COVID-19 in ICU patients are lacking. One study that evaluated the use of ascorbic acid in COVID-19 patients was conducted by Jing et al. They randomized patients admitted to the ICU with COVID-19 to receive high dose ascorbic acid $12 \mathrm{~g}$ every 12 hours for seven days versus placebo. This study showed no benefit of using ascorbic acid in the 28-days mortality or duration of mechanical ventilation. However, oxygenation was significantly improved in the ascorbic acid patients (28). A recent RCT was stopped after interim analysis due to futility, high-dose zinc 
and vitamin C (ascorbic acid) had no impact on the course of symptoms in patients with mild COVID-19 but did not evaluate the benefits in critically ill COVID 19. In addition, there was no difference in secondary endpoints, including days to symptom resolution, severity of symptoms, hospitalizations, or deaths ${ }^{34}$.

Our study shows that low supplemental dose of enteral ascorbic acid had a significant reduction in thrombosis risk during ICU stay. The underlying benefit of ascorbic acid on thrombosis could be due to its anti-inflammatory properties. Of interest, deep vein thrombosis (DVT) prophylaxis considered as a standard of care in our patients, and using DVT prophylaxis was similar between the two groups ( $p$ value: $>0.999$ ). Several published studies showed that the incidence of thrombosis was high in critically ill patients with COVID-19 $(31,33)$. Several pharmacological regimens have been proposed to impact the outcomes for patients with COVID-19 positively. Out of these regimens, only dexamethasone has been shown to improve survival (15). None of the previously investigated pharmacological modalities for COVID-19 have shown a reduction in the risk of thrombosis.

Our findings showed a statistically insignificant higher rate of AKI, liver Injury and respiratory failure requiring MV in ascorbic acid group. Even though multiple trials showed positive outcomes with ascorbic acid $(8,20,21,24,25)$, other trials did not show an improvement in the clinical outcomes ${ }^{(26,27)}$. In a phase I trial, 16 patients with severe sepsis received a high IV ascorbic acid dose (50-200 $\mathrm{mg} / \mathrm{kg} /$ day) for four days. Ascorbic acid use showed a reduction in sequential organ failure assessment (SOFA) score and proinflammatory biomarkers while being well-tolerated (7). Nathens et al. used IV ascorbic acid $1 \mathrm{~g}$ every eight hours for 28 days in 594 critically ill surgical patients and found a significantly lower incidence of multi-organ failure, shorter mechanical ventilation duration, and ICU length of stay (22). The lack of significant improvement in the clinical outcomes in our study could be related to the use of supplemental dose which is lower dose in comparison to previous published studies.

Ascorbic acid in non-COVID-19 patients has been studied extensively in several randomized controlled trials and observational studies with mixed results due to the lack of consistency in terms of the ascorbic acid dose, route, timing, frequency of administration in these studies, and primary outcome measures. Studies addressing ascorbic acid in COVID 19 critically ill patients are lacking. Our study provides a hypothesis-generating idea of the potential benefit of using ascorbic acid in critically ill patients with COVID-19 in reducing the risk of thrombosis. We believe that this hypothesis needs to be further investigated at a larger scale using more robust, validated modalities and study designs to eliminate the risk of bias.

Our study has several limitations in terms of the retrospective design and the heterogeneity in the comorbid conditions and disease severity; that was minimized via using the propensity score. Also, baseline ascorbic acid levels were not measured before initiating the supplemental regimen, given our study's retrospective nature. Moreover, there was a dynamic change in the clinical practice of managing patients with COVID-19 as evidence continued to emerge over time. Furthermore, there was no consensus on when to start ascorbic acid, and it was mainly at the discretion of the treating team. 


\section{Conclusion}

The use of ascorbic acid was not assosiated with in-hospital nor 30-days ICU mortality reduction. Using supplemental ascorbic acid doses as an adjunctive therapy in critically ill patients with COVID-19 was associated with lower incidence of thrombosis. Further studies are warranted to confirm these findings.

\section{Abbreviations}

Intensive care units (ICUs), Coronavirus disease (COVID-19), Mechanical ventilation (MV), Length of Stay (LOS).

\section{Declarations}

\section{Acknowledgements}

Not applicable.

\section{Author contributions}

All authors contributed to data collections, analysis, drafted, revised, and approved the final version of the manuscript.

\section{Funding}

None.

\section{Availability of data and material}

The datasets used and/or analyzed during the current study are available from corresponding author on reasonable request.

\section{Ethics approval and consent to participate}

The study was approved on November 19th, 2020, by King Abdullah International Medical Research Center (KAIMRC)-Institutional Review Board (IRB), Riyadh, Saudi Arabia (Reference No: RC20/589/R). All methods were performed in accordance with relevant guidelines and regulations.

Participants' confidentiality was strictly observed throughout the study by using anonymous unique serial number for each subject and restricting data only to the investigators. KAIMRC-IRB committee waived the informed consent due to its retrospective nature.

\section{Consent for publication}

Not applicable. 


\section{Competing interests}

No author has a conflict of interest in this study.

\section{Author details}

${ }^{1}$ Pharmaceutical Care Department, King Abdulaziz Medical City, Riyadh, Saudi Arabia

${ }^{2}$ Department of Pharmacy Practice, Faculty of Pharmacy, King Abdulaziz University, Jeddah, Saudi Arabia

${ }^{3}$ Pharmaceutical Care Department, King Abdulaziz University Hospital, Jeddah, Saudi Arabia

${ }^{4}$ College of Medicine, King Saud Bin Abdulaziz University for Health Sciences, King Abdullah International Medical Research Center

${ }^{5}$ Intensive Care Department, King Abdulaziz Medical City, Riyadh, Saudi Arabia

${ }^{6}$ College of Pharmacy, King Saud bin Abdulaziz University for Health Sciences, Riyadh, Saudi Arabia.

${ }^{7}$ King Abdullah International Medical Research Center, Biostatistics and Bioinformatics Department, Riyadh, Saudi Arabia.

${ }^{8}$ Department of Clinical Pharmacy, College of Pharmacy, King Saud University, Riyadh, Saudi Arabia.

\section{References}

1. Huang C, Wang Y, Li X, et al. Clinical features of patients infected with 2019 novel coronavirus in Wuhan, China. Lancet. 2020;395(10223):497-506

2. Carr AC, Maggini S. Vitamin C and Immune Function. Nutrients. (2017) 9:1211.

3. Leibovitz B, Siegel BV. Ascorbic acid and the immune response. Adv Exp Med Biol. (1981) 135:1-25.

4. Dey S, Bishayi B. Killing of $S$. aureus in murine peritoneal macrophages by Ascorbic acid along with antibiotics Chloramphenicol or Ofloxacin: correlation with inflammation. Microb Pathog. (2018) 115:239-50.

5. Wei XB, Wang ZH, Liao XL, et al. Efficacy of vitamin C in patients with sepsis: an updated metaanalysis. Eur J Pharmacol. 2020;868:172889.

6. Fisher BJ, Seropian IM, Kraskauskas D, et al. Ascorbic acid attenuates lipopolysaccharide-induced acute lung injury. Crit Care Med. 2011;39(6):1454-1460.

7. Fowler AA, 3rd, Syed AA, Knowlson S, et al. Phase I safety trial of intravenous ascorbic acid in patients with severe sepsis. J Trans/ Med. 2014;12:32.

8. Fowler AA, 3rd, Truwit JD, Hite RD, et al. Effect of vitamin C infusion on organ failure and biomarkers of inflammation and vascular injury in patients with sepsis and severe acute respiratory failure: the CITRIS-ALI randomized clinical trial. JAMA. 2019;322(13):1261-1270. 
9. Marik PE, Khangoora V, Rivera R, Hooper MH, Catravas J. Hydrocortisone, vitamin C, and thiamine for the treatment of severe sepsis and septic shock: a retrospective before-after study. Chest. 2017;151(6):1229-1238.

10. Kim WY, Jo EJ, Eom JS, et al. Combined vitamin C, hydrocortisone, and thiamine therapy for patients with severe pneumonia who were admitted to the intensive care unit: propensity score-based analysis of a before-after cohort study. J Crit Care. 2018;47:211-218.

11. Hemilä H, Chalker E. Vitamin C Can Shorten the Length of Stay in the ICU: A Meta-Analysis. Nutrients. 2019 Mar 27;11(4):708.

12. H.C. Gorton, K. Jarvis. The effectiveness of vitamin $\mathrm{C}$ in preventing and relieving the symptoms of virus-induced respiratory infections. J Manipulative Physiol Ther, 22 (1999), pp. 530-533

13. Kim TK, Lim HR, Byun JS.Vitamin C supplementation reduces the odds of developing a common cold in Republic of Korea Army recruits: randomized controlled trial. BMJ Mil Health Published Online First: 05 March 2020. doi: 10.1136/bmjmilitary-2019-001384

14. Richard Z. Cheng. Can early and high intravenous dose of vitamin $C$ prevent and treat coronavirus disease 2019 (COVID-19)?. Medicine in Drug Discovery,Volume 5,2020, 100028, ISSN 25900986,https://doi.org/10.1016/j.medidd.2020.100028.

15. Recovery Collaborative Group, Horby P, Lim WS, et al. Dexamethasone in hospitalized patients with COVID-19 - preliminary report. N Engl J Med. 2020.

16. A.C. Carr, P.C. Rosengrave, S. Bayer, S. Chambers, J. Mehrtens, G.M. Shaw Hypovitaminosis $C$ and vitamin $\mathrm{C}$ deficiency in critically ill patients despite recommended enteral and parenteral intakes Crit Care, 21 (2017).

17. Borrelli E., Roux-Lombard P., Grau G.E., Giradin E., Ricou B., Dayer J., Suter P.M. Plasma concentrations of cytokines, their soluble receptors, and antioxidant vitamins can predict the development of multiple organ failure in patients at risk. Crit. Care Med. (1996).

18. Grooth HJ, Spoelstra-de Man AME, Oudemans-van Straaten HM. Early plasma vitamin C concentration, organ dysfunction and ICU mortality. Intensive Care Med (2014).

19. Li R, Guo C, Li Y, Qin Z, Huang W. Therapeutic targets and signaling mechanisms of vitamin C activity against sepsis: a bioinformatics study. Brief Bioinform. 2020.

20. Peters E, Goetzsche J, Grobbelaar B, Noakes T. Vitamin C supplementation reduces the incidence of postrace symptoms of upper-respiratory-tract infection in ultramarathon runners. Am J Clin Nutr. (1993)

21. Hunt C, Chakravorty N, Annan G, Habibzadeh N, Schorah C. The clinical effects of vitamin C supplementation in elderly hospitalised patients with acute respiratory infections. Int J Vitam Nutr Res. (1994)

22. A.B. Nathens, M.J. Neff, G.J. Jurkovich, et al. Randomized, prospective trial of antioxidant supplementation in critically ill surgical patients Ann Surg, 236 (2002)

23. Marik PE, Khangoora V, Rivera R, Hooper MH, Catravas J. Hydrocortisone, Vitamin C, and Thiamine for the Treatment of Severe Sepsis and Septic Shock: A Retrospective Before-After Study. Chest. 
(2017)

24. Zabet MH, Mohammadi M, Ramezani M, Khalili H. Effect of high-dose Ascorbic acid on vasopressor's requirement in septic shock. J Res Pharm Pract. 2016;5:94-100

25. Nabil Habib T, Ahmed I. Early adjuvant intravenous vitamin $C$ treatment in septic shock may resolve the vasopressor depend- ence. Int J Microbiol Adv Immunol. (2017)

26. Fujii T, Luethi N, Young P, Frei D, Eastwood G, French C, et al. Effect of vitamin C, hydrocortisone, and thiamine vs hydrocortisone alone on time alive and free of vaso- pressor support among patients with septic shock. JAMA. (2020)

27. Yoo J, Kim R, Ju S, Lee S, Cho Y, Jeong Y, et al. Clinical impact of supplementation of vitamins B1 and $C$ on patients with sepsis-related acute respiratory distress syndrome. Tuberc Respir Dis. (2020)

28. Jing Zhang, Xin Rao, Yiming Li, et al. Pilot trial of high-dose vitamin C in critically ill COVID-19 patients. Ann Intensive Care. (2021)

29. Lin CY, Chen YC. Acute kidney injury classification: AKIN and RIFLE criteria in critical patients. World J Crit Care Med. 2012;1(2):40-45. Published 2012 Apr 4. doi:10.5492/wjccm.v1.i2.40

30. ICD - ICD-10-CM - International Classification of Diseases, Tenth Revision, Clinical Modification. Cdc.gov. https://www.cdc.gov/nchs/icd/icd10cm.htm. Published 2021. Accessed January 29, 2021.

31. Al Sulaiman K, Al Juhani O, Eljaaly K et al. Clinical Features and Outcomes of Critically III Patients with Coronavirus Disease 2019 (COVID-19): A Multicenter Cohort Study. International Journal of Infectious Diseases. 2021. doi:10.1016/j.ijid.2021.02.037

32. Donnino MW, Andersen LW, Chase M, et al. Randomized, Double-Blind, Placebo-Controlled Trial of Thiamine as a Metabolic Resuscitator in Septic Shock: A Pilot Study. Crit Care Med. 2016;44(2):360367. doi:10.1097/CCM.0000000000001572

33. Klok FA, Kruip MJHA, van der Meer NJM, et al. Incidence of thrombotic complications in critically ill ICU patients with COVID-19. Thromb Res. 2020;191:145-147. doi:10.1016/j.thromres.2020.04.013

34. Thomas S, Patel D, Bittel B, et al. Effect of High-Dose Zinc and Ascorbic Acid Supplementation vs Usual Care on Symptom Length and Reduction Among Ambulatory Patients With SARS-CoV-2 Infection: The COVID A to Z Randomized Clinical Trial. JAMA Netw Open. 2021;4(2):e210369.

\section{Tables}

Table 1 Regression analysis for the outcomes 


\begin{tabular}{|c|c|c|c|c|c|}
\hline \multirow[t]{2}{*}{ Outcomes } & \multicolumn{2}{|c|}{$\begin{array}{c}\text { Ascorbic Acid group } \\
\mathrm{n} \text { of outcomes/Total no-of } \\
\text { patients }\end{array}$} & \multirow[b]{2}{*}{ P-value } & \multirow[t]{2}{*}{ Odds Ratio (OR) (95\%CI) } & \multirow[t]{2}{*}{ P-value } \\
\hline & NO & Yes & & & \\
\hline \multicolumn{6}{|l|}{ In-hospital mortality, n (\%) $\Delta$} \\
\hline Analysis on all eligible patients & $\begin{array}{l}275 / 558 \\
(49.3) \\
\end{array}$ & $50 / 149(33.6)$ & $0.0006^{\wedge} \wedge$ & $0.50(0.330,0.759)$ & $0.0011^{* \wedge}$ \\
\hline Propensity score matched & $59 / 142(41.6)$ & $46 / 142(32.4)$ & $0.11^{\wedge} \wedge$ & $0.77(0.476,1.234)$ & $0.27 \$$ \\
\hline \multicolumn{6}{|l|}{ ICU mortality within 30 days $\Delta$} \\
\hline Analysis on all eligible patients & $\begin{array}{l}235 / 540 \\
(43.5) \\
\end{array}$ & $40 / 146(27.4)$ & $\begin{array}{l}0 . \\
0004^{\wedge} \wedge\end{array}$ & $0.51(0.332,0.794)$ & $0.0027^{* \wedge}$ \\
\hline \multirow[t]{2}{*}{ Propensity score matched } & $48 / 136(35.3)$ & $37 / 139(26.6)$ & $0.11^{\wedge} \wedge$ & $0.73(0.438,1.204)$ & $0.21 \$$ \\
\hline & & & & $\begin{array}{l}\text { beta coefficient } \\
\text { (Estimates) }(95 \% \mathrm{CI})\end{array}$ & P-value \\
\hline $\begin{array}{l}\text { MV duration during ICU stay Days, } \\
\text { Median (IQR) \& }\end{array}$ & $\begin{array}{l}3.0(1.00- \\
11.50) \\
\end{array}$ & $3(0.00-12.00)$ & $0.49^{\wedge} \wedge$ & $0.14(-0.24,0.52)$ & 0.47 \$* \\
\hline $\begin{array}{l}\text { ICU Length of Stay Days, Median } \\
\text { (IQR) \& }\end{array}$ & $\begin{array}{l}7.0(4.00- \\
12.00)\end{array}$ & $\begin{array}{l}8.5(5.00- \\
15.00)\end{array}$ & $0.26^{\wedge} \wedge$ & $0.47(0.26,0.68)$ & $\begin{array}{r}<.0001 \\
\$^{*}\end{array}$ \\
\hline $\begin{array}{l}\text { Hospital Length of Stay Days, Median } \\
\text { (IQR) \& }\end{array}$ & $\begin{array}{l}13.5(10.00- \\
23.00)\end{array}$ & $\begin{array}{l}17.0(12.00- \\
27.00)\end{array}$ & $0.05^{\wedge} \wedge$ & $0.73(0.51,0.95)$ & $\begin{array}{r}<.0001 \\
\$^{*}\end{array}$ \\
\hline \multicolumn{6}{|c|}{$\begin{array}{l}\Delta \text { Denominator of the percentage is the total number of patients } \\
\text { \& Denominator is patients who survived. } \\
\text { *T -Test / ^ Wilcoxon rank sum test is used to calculate the P-value. } \\
\wedge^{\wedge} \text { Chi-square test is used to calculate the P-value. } \\
\text { **Fisher Exact test is used to calculate the P-value. } \\
\$ * \text { propensity score adjusted Generalized linear model is used to calculate estimates and p-value. } \\
\$ \text { propensity score adjusted Logistic regression is used to calculate Odds ratio and p-value. } \\
{ }^{*} \text { Multivariate Logistic regression is used after adjusting for patient's baseline severity scores, systemic use of corticosteroids } \\
\text { and hospital center to calculate Odds ratio and p-value. }\end{array}$} \\
\hline
\end{tabular}

Table 2 Regression analysis for ICU complication (s) during ICU stay 


\begin{tabular}{|c|c|c|c|c|c|}
\hline \multirow[t]{2}{*}{ Outcomes } & \multicolumn{2}{|c|}{$\begin{array}{c}\text { Ascorbic Acid group } \\
\mathrm{n} \text { of outcomes/Total no-of patients }\end{array}$} & \multirow[t]{2}{*}{ P-value } & \multirow[t]{2}{*}{ OR/Estimates (95\%CI) } & \multirow[t]{2}{*}{ P-value $\$$} \\
\hline & No & Yes & & & \\
\hline \multicolumn{6}{|l|}{ Acute Kidney Injury (AKI), n (\%) } \\
\hline Analysis on all eligible patients & $277 / 570(48.6)$ & $58 / 156(37.2)$ & $0.01^{\wedge} \wedge$ & $0.66(0.444,0.984)$ & $0.04^{* \wedge}$ \\
\hline Propensity score matched & $51 / 146(34.9)$ & $56 / 148(37.8)$ & $0.60^{\wedge \wedge}$ & $1.34(0.837,2.150)$ & $0.22^{\$}$ \\
\hline \multicolumn{6}{|l|}{ Liver Injury, n (\%) } \\
\hline Analysis on all eligible patients & $63 / 568(11.1)$ & $14 / 156(9.0)$ & $0.44^{\wedge} \wedge$ & $0.52(0.277,0.989)$ & $0.04^{* \wedge}$ \\
\hline Propensity score matched & $9 / 146(6.2)$ & $13 / 148(8.8)$ & $0.39^{\wedge} \wedge$ & $1.17(0.517,2.653)$ & $0.70^{\$}$ \\
\hline \multicolumn{6}{|l|}{ Respiratory Failure Required MV, n (\%) \$ } \\
\hline Analysis on all eligible patients & $35 / 156(22.4)$ & $27 / 83(32.5)$ & $0.12^{\wedge}$ & $0.97(0.51,1.82)$ & $0.93^{* \wedge}$ \\
\hline Propensity score matched & $34 / 72(47.2)$ & $25 / 48(52.0)$ & $0.71^{\wedge \wedge}$ & $1.05(0.51,2.14)$ & $0.90^{\$}$ \\
\hline \multicolumn{6}{|l|}{ Thrombosis During ICU, $\mathrm{n}(\%)$} \\
\hline Analysis on all eligible patients & $64 / 565(11.3)$ & $9 / 5.8(2.3)$ & $0.04^{\wedge}$ & $0.35(0.167,0.717)$ & $0.0043^{* \wedge}$ \\
\hline Propensity score matched & $19 / 146(13.0)$ & $9 / 147(6.1)$ & $0.04^{\wedge} \wedge$ & $0.42(0.184,0.937)$ & $0.03^{\$}$ \\
\hline \multicolumn{6}{|c|}{$\begin{array}{l}\text {-Denominator of the percentage is the total number of patients. } \\
\text { OR: Odds Ratio } \\
* \text { T -Test / ^ Wilcoxon rank sum test is used to calculate the P-value. } \\
\wedge \wedge \text { Chi-square /**Fisher Exact test is used to calculate the P-value. } \\
\$ \text { propensity score adjusted Logistic regression is used to calculate Odds ratio and p-value. } \\
* \wedge \text { Multivariate Logistic regression is used after adjusting for patient's baseline severity scores, systemic use of corticosteroids } \\
\text { and hospital center to calculate Odds ratio and p-value } \\
\text { \$ Denominator of the percentage is non-mechanically ventilated patients with } 24 \text { hours of ICU admission. }\end{array}$} \\
\hline
\end{tabular}

\section{Figures}




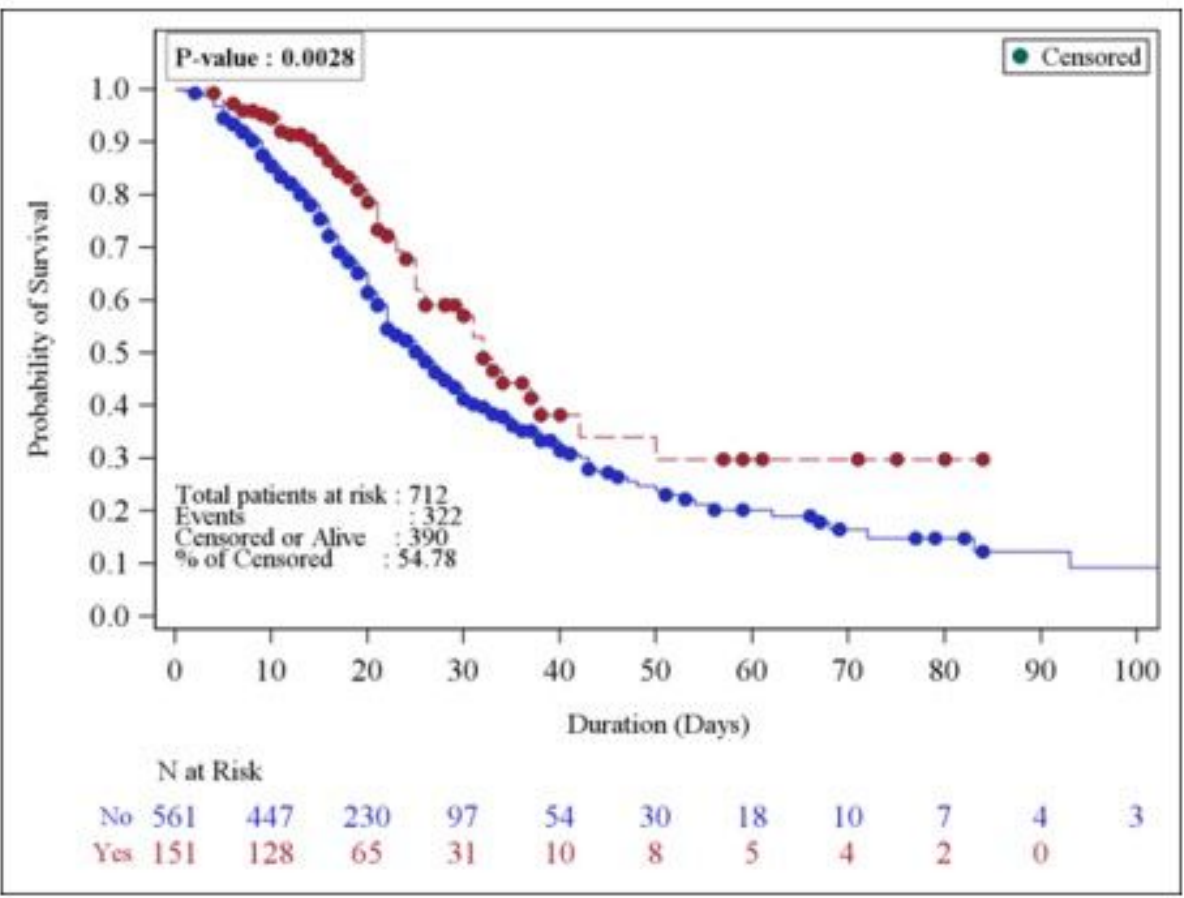

a

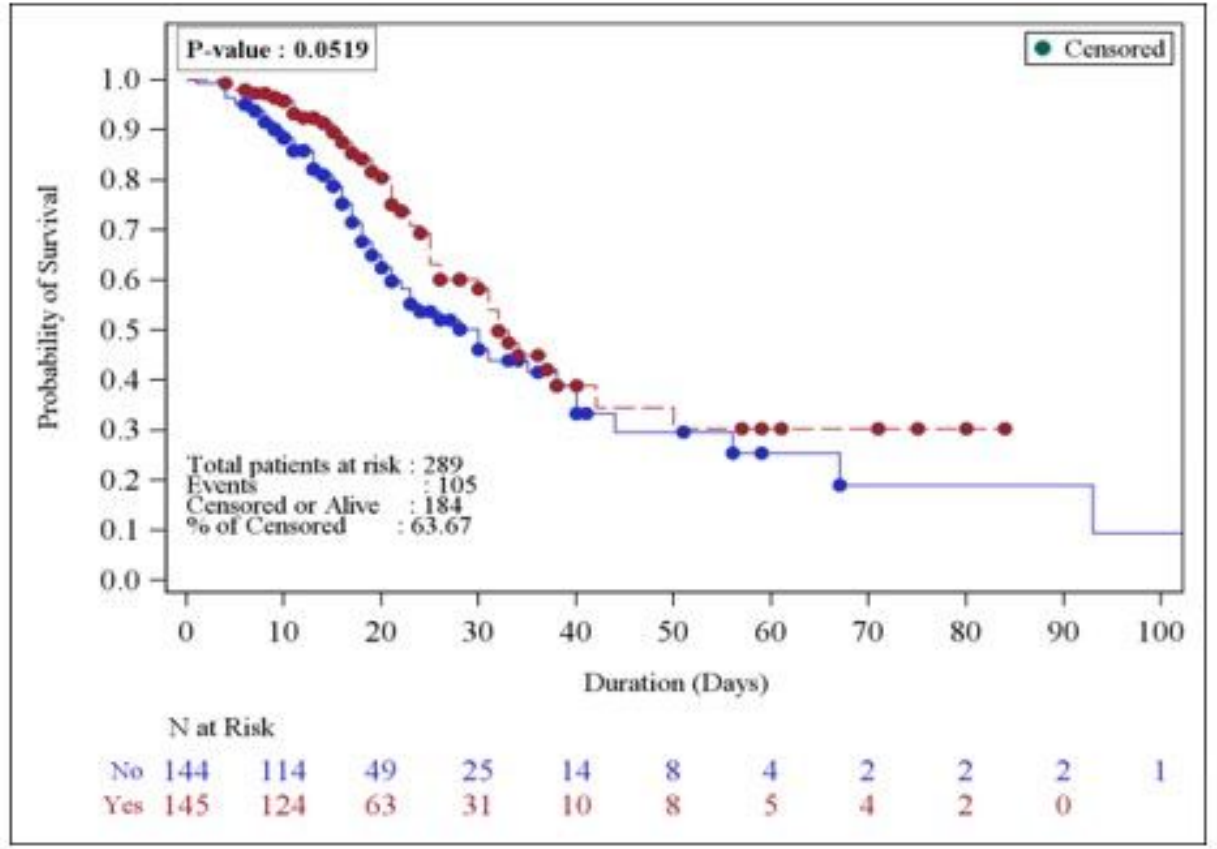

b

Figure 1

a. Time to hospital discharge in patients received ascorbic acid and those who did not (All cohort). b. Time to hospital discharge in propensity score matched patients who received ascorbic acid and those who did not

\section{Supplementary Files}


This is a list of supplementary files associated with this preprint. Click to download.

- Additionalfile1DemographyandBaselinecharacteristics.docx

- Additionalfile2Coexistingillness.docx 